\title{
छூ \\ New method of calculating the wakefields of a point charge in a waveguide of arbitrary cross section
}

\author{
S. S. Baturin* and A. D. Kanareykin \\ Euclid Techlabs, LLC, Solon, Ohio 44139, USA \\ and St. Petersburg Electrotechnical University LETI, St. Petersburg, Russia 197376
}

(Received 21 March 2016; published 23 May 2016)

\begin{abstract}
A new method for calculating the Cherenkov wakefield acting on a point charged particle passing through a longitudinally homogeneous structure lined with layer(s) of an arbitrary retarding (dielectric, resistive, or corrugated) material has been developed. In this paper we present a rigorous derivation of the expressions for the fields that are valid at the cross section of the particle on the basis of a conformal mapping method. This new formalism allows reduction of the loss factor calculation to a simple derivation of a conformal mapping function from the arbitrary cross section onto a circular disc. We generalize these results to the case of a bunch with an arbitrary transverse distribution by deriving a two-dimensional Green function at the cross section of the particle. Consequently, for the first time analytical expressions for the transverse distributions of the electric field $E_{z}$ for the most commonly used cylindrical, planar and elliptical cross section geometries are found. The proposed approach significantly decreases simulation time and opens new possibilities in optimizing wakefield effects resulting from short charged particle bunches for FEL and Linear Collider applications.
\end{abstract}

DOI: 10.1103/PhysRevAccelBeams.19.051001

\section{INTRODUCTION}

Wakefields excited when very short bunches pass through accelerating structures or other longitudinally extended components of a beam line (pipes, collimators, bellows), are of major concern for linear colliders (ILC [1], CLIC [2]), and FEL (LCLS-II, X-FEL, etc.) $[3,4]$ and other accelerator projects. A particular example is the need to quantitatively understand the wakefields generated by short bunches while passing through the small apertures of undulator vacuum chambers in X-ray FELs. These accelerators are currently being developed to achieve high brightness X-ray photon beams with extremely narrow bandwidth. The parameters require precise control of energy spread, emittance and transverse stability of electron bunches that are affected by the wakefields generated by these bunches in the beamline components. Therefore, analysis of the short-range wakefields in the longitudinally extended components, especially in undulator vacuum chambers, is needed to control the energy spread and emittance of the short bunches [5,6].

Theoretical analysis of Cherenkov radiation commonly considers a "short bunch" approach [7-10]. This can be applied for various Cherenkov generation parameters, where the moving bunch size is much less than the

*s.s.baturin@gmail.com

Published by the American Physical Society under the terms of the Creative Commons Attribution 3.0 License. Further distribution of this work must maintain attribution to the author $(s)$ and the published article's title, journal citation, and DOI. fundamental wavelength if the high frequency spectral range is not under investigation. Wakefields generated by short bunches at commonly used (and other) shapes of the accelerator component cross sections have been considered in many publications. Circular, planar and more complicated elliptical and rectangular cross sections are currently of special interest. For example, a possible choice for a small-gap vacuum chamber is one with an elliptical cross section, often approximated by a planar or circular geometry depending on the elliptical aspect ratio.

The loss factor for a cylindrical geometry for a bunch with arbitrary displacement from the structure axis can be found elsewhere [11]. The $E_{z}$ field component of a planar waveguide has been studied in many publications, for instance Ref. [7]. A rectangular structure with two retardation layers was initially considered in $[12,13]$ and later in [14], where it was analyzed using the transverse operator to develop a rigorous full solution for the wakefields. Elliptical geometries were also analyzed; see [15] and references therein.

The basic analytic solution technique used the references listed above was the mode decomposition method, where electromagnetic fields for a vacuum channel and surrounding polarizable materials were found separately in the frequency domain, and then matched using boundary conditions on the interface between them. Finally, the inverse Fourier transformation is applied and the problem is solved. The main limitation of this method is that each solution is obtained for a particular class of geometries. In addition, the final solution is represented in terms of infinite series that need to be truncated for practical calculations. In 
case of very short bunches, this series reduction has to be done taking in account a large number of terms to achieve reasonable accuracy for the wakefield amplitude inside the bunch. These two limitations bound the application range of the previously obtained analytical solutions and reduce the opportunity for geometry optimization. In addition, the large number of modes enormously increases the simulation time required for beam break up (BBU) computations.

A new theoretical approach that can be used for obtaining direct analytical formulas for electromagnetic field components at the position of a point particle was recently developed [16]. It was demonstrated that the longitudinal part of the Lorentz force acting on the point particle does not depend on the polarizing layer geometry (corrugation) or material properties (dielectric, resistive wall) of the waveguide, and that it is a constant for any given transverse dimensions or cross-sectional shape of the structure. The equivalence and exact matching of the longitudinal electric fields $E_{z}$ of beams passing through various waveguide configurations was also analyzed. The approach uses an integral relation based on the cylindrical slow-wave structure model. It was shown that for the planar, square, and other cross section geometries, one can obtain a corresponding form factor coefficient by using a conformal mapping of these shapes onto the disk, Fig. 1(a) $[16,17]$. This approach was later extended to the case of surface impedance (Leontovich) boundary conditions [18].

However, the question of how to calculate the transverse distribution of the fields and Lorentz force in the cross section of the structure where the particle is located was not examined in detail. The method described in [16] allows us to calculate the longitudinal electric field $E_{z}$ and longitudinal derivative of the transverse part of the Lorentz force at the point of the particle, which is also assumed to be pointlike. In this paper we extend the idea of [16] to a bunch which is a pointlike only in the longitudinal coordinate but has some arbitrary distribution in the transverse coordinates. We will obtain analytical expressions giving the dependence of the longitudinal fields $E_{z}, H_{z}$ and the transverse part of the Lorentz force in the cross section of a particle on the transverse coordinates. Analytical formulas obtained here are actually the Green functions and therefore can be applied to the field calculations of bunches distributed along the transverse coordinates. We especially consider here the most important cases of commonly used cross section geometries and verify the results numerically using previously developed methods. The proposed approach allows deriving simple analytical expressions that significantly reduce simulation time and generalize to arbitrary cross section shapes that, in turn, open new possibilities for high accuracy analysis of wakefields resulting from short charged particle bunches for FEL and Linear Collider applications.

A few words about the terminology used here. All fields considered in this paper are calculated in the plane where the point charge is positioned at the time $t$ but not behind the bunch. At the same time these fields cannot be called "self-fields" that are usually defined as the fields associated with the moving charge but not the radiation fields [19]. The longitudinal field components we consider in this paper are radiation fields. It is also important that the longitudinal components of the self-fields are equal to zero for ultrarelativistic particle, while the radiation components are caused by the presence of boundaries (waveguide walls in our case) that terminate the fields of the moving bunch. By commonly accepted terminology these radiation fields are called wakefields. Therefore, even if these fields are located strictly in the plane of the moving point charge the "wakefields" terminology is correct and needs to be used.

The paper is organized as follows. In Sec. II we derive the main formula for the transverse coordinate dependence of the longitudinal field components $E_{z}$ and $H_{z}$ on the cross section of a point particle. This expands the method previously introduced [16] based only on the assumption that the electromagnetic field in the cross section of a particle is equal to zero outside the vacuum channel. In Sec. III the transverse part of the Lorentz force is considered acting on a particle moving along a vacuum channel of arbitrary cross-sectional shape. The expression obtained is

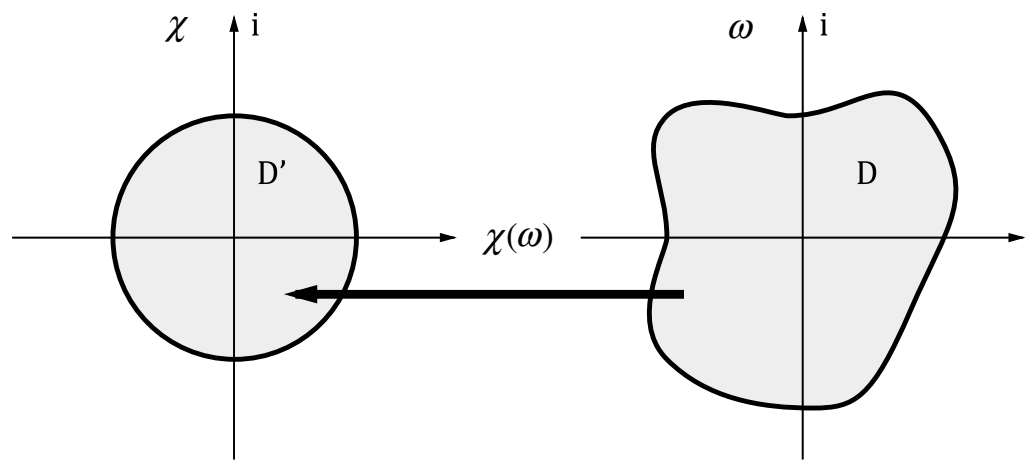

(a)

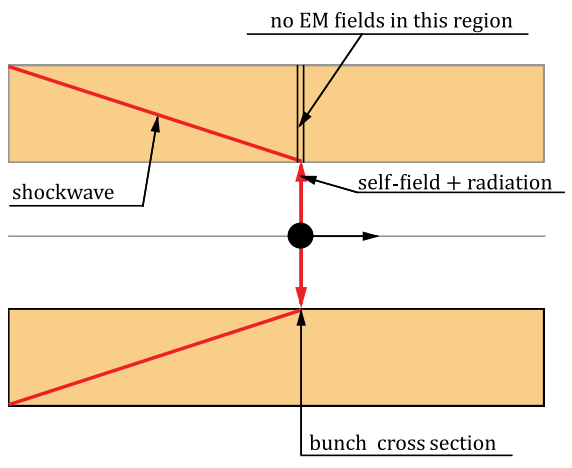

(b)

FIG. 1. (a) Definition of the conformal map from the $\omega$ plane to the $\chi$ plane. (b) Schematic of the shock wave formation process. 
the two-dimensional Green function. The relativistic Gauss theorem [16] is presented in Sec. IV derived with the use of the formalism demonstrated in Sec. II. In Sec. V, we consider case studies of transverse distribution of the $E_{z}$ field component for the most commonly used cross section geometries: cylindrical, planar, and rectangular waveguides. The latter is considered with the polarizing material layers on the top and bottom, and with perfectly conducting side walls. Finally, special attention is paid to the elliptical cross section shape, Sec. V D, as an important geometry for the wakefield analysis of FEL undulators. Short range longitudinal and transverse wakefunctions estimations are presented in Appendices A and B correspondingly.

\section{GENERAL APPROACH}

In this section, we derive the main formula for the transverse distribution of the longitudinal fields $E_{z}$ and $H_{z}$ at the cross section of a structure normal to its axis and containing the point particle. The particle is assumed to be moving parallel to the structure axis and to be two dimensional. The method is based only on the assumption that the complete electromagnetic field in the cross section of a waveguide containing the particle is equal to zero outside the vacuum channel, which is a simple consequence of the fact that the phase velocity of the Cherenkov radiation in the waveguide retarding layers (defined by dielectric, corrugation or resistive properties) is less than the speed of light; the particle is assumed to be relativistic, Fig. 1(b).

Let us consider the Maxwell system in cgs units inside the vacuum channel:

$$
\begin{aligned}
\nabla \times \mathbf{E} & =-\frac{1}{c} \frac{\partial \mathbf{H}}{\partial t}, \\
\nabla \times \mathbf{H} & =\frac{4 \pi}{c} \mathbf{j}+\frac{1}{c} \frac{\partial \mathbf{E}}{\partial t}, \\
\nabla \cdot \mathbf{E} & =4 \pi \rho, \\
\nabla \cdot \mathbf{H} & =0 .
\end{aligned}
$$

We assume that the particle is moving along the $\mathrm{z}$-axis of the structure and that the structure is longitudinally homogeneous. In the ultrarelativistic limit the current that produces the particle and its charge density can be written as:

$$
\begin{aligned}
& j=j_{z}=c \rho, \\
& \rho=Q \delta\left(x-x_{0}\right) \delta\left(y-y_{0}\right) \delta(z-c t) .
\end{aligned}
$$

Let us introduce a new coordinate $\zeta=c t-z$ and split the Maxwell system into two parts:

$$
\begin{aligned}
& {[\nabla \times \mathbf{E}]_{\perp}=-\frac{\partial \mathbf{H}_{\perp}}{\partial \zeta},} \\
& {[\nabla \times \mathbf{H}]_{\perp}=\frac{\partial \mathbf{E}_{\perp}}{\partial \zeta}}
\end{aligned}
$$

and

$$
\begin{aligned}
\nabla_{\perp} \times \mathbf{E}_{\perp} & =-\frac{\partial H_{z}}{\partial \zeta} \\
\nabla_{\perp} \times \mathbf{H}_{\perp} & =4 \pi \rho+\frac{\partial E_{z}}{\partial \zeta} \\
\nabla_{\perp} \cdot \mathbf{E}_{\perp} & =4 \pi \rho+\frac{\partial E_{z}}{\partial \zeta} \\
\nabla_{\perp} \cdot \mathbf{H}_{\perp} & =\frac{\partial H_{z}}{\partial \zeta}
\end{aligned}
$$

Here the $\perp$ symbol indicates field components orthogonal to the $z$-axis and $\nabla_{\perp}$ is the nabla operator in the plane orthogonal to the $z$-axis. The equations (3) can be reduced to:

$$
\begin{aligned}
\frac{\partial H_{z}}{\partial x} & =\frac{\partial E_{z}}{\partial y}, \\
\frac{\partial H_{z}}{\partial y} & =-\frac{\partial E_{z}}{\partial x} .
\end{aligned}
$$

Equation (5) could be considered as the Cauchy-Riemann equations for some function defined in the complex plane. Taking the waveguide vacuum channel cross section which includes the particle, as the complex plane, (4) can be rewritten as

$$
\begin{aligned}
& \nabla_{\perp} \cdot \mathbf{E}_{\perp}+i \nabla_{\perp} \times \mathbf{E}_{\perp}=4 \pi \rho+\frac{\partial E_{z}}{\partial \zeta}-i \frac{\partial H_{z}}{\partial \zeta} \\
& \nabla_{\perp} \cdot \mathbf{H}_{\perp}+i \nabla_{\perp} \times \mathbf{H}_{\perp}=i\left(4 \pi \rho+\frac{\partial E_{z}}{\partial \zeta}-i \frac{\partial H_{z}}{\partial \zeta}\right) .
\end{aligned}
$$

We introduce the complex functions

$$
\begin{aligned}
& e=E_{x}+i E_{y}, \\
& h=H_{x}+i H_{y} .
\end{aligned}
$$

We introduce the operator [20]

$$
\nabla_{c}=\frac{\partial}{\partial x}+i \frac{\partial}{\partial y}
$$

One can see that

$$
\begin{aligned}
& \nabla_{c}^{*} e=\nabla_{\perp} \cdot \mathbf{E}_{\perp}+i \nabla_{\perp} \times \mathbf{E}_{\perp}, \\
& \nabla_{c}^{*} h=\nabla_{\perp} \cdot \mathbf{H}_{\perp}+i \nabla_{\perp} \times \mathbf{H}_{\perp} .
\end{aligned}
$$

Here the ${ }^{*}$ - symbol means complex conjugation. On the other hand one can rewrite (8) as 


$$
\begin{aligned}
& \nabla_{c}=2 \frac{\partial}{\partial \chi^{*}}, \\
& \nabla_{c}^{*}=2 \frac{\partial}{\partial \chi}
\end{aligned}
$$

where $\chi=x+i y$. From (5) one can then treat the function $L=\frac{\partial E_{z}}{\partial \zeta}-i \frac{\partial H_{z}}{\partial \zeta}$ as a plane vector field. According to [21], since this field has no sources and vortices, it could be considered as a derivative of a complex potential

$$
\frac{\partial g_{Z}}{\partial \chi}=\frac{\partial E_{z}}{\partial \zeta}-i \frac{\partial H_{z}}{\partial \zeta}
$$

We define the Fourier transformation as:

$$
\begin{aligned}
\tilde{f}(k) & =\frac{1}{2 \pi} \int_{-\infty}^{\infty} f(\zeta) \exp (-i k \zeta) d \zeta \\
f(\zeta) & =\int_{-\infty}^{\infty} \tilde{f}(k) \exp (i k \zeta) d \zeta
\end{aligned}
$$

Fourier transform of a derivative is given by

$$
\frac{1}{2 \pi} \int \frac{d f(\zeta)}{d \zeta} \exp (-i k \zeta) d \zeta=\frac{i k}{2 \pi} \tilde{f}(k)
$$

Performing a Fourier transformation on Eq. (11),

$$
\frac{\partial \tilde{g}_{Z}}{\partial \chi}=i k\left(\tilde{E}_{z}-i \tilde{H}_{z}\right)
$$

and by combining (6), (10), (7), and (14), we obtain

$$
2 \frac{\partial \tilde{e}}{\partial \chi}=4 \pi \tilde{\rho}+\frac{\partial \tilde{g}_{Z}}{\partial \chi}
$$

Since $h=i e$, we need to consider only the equation for $e$.

Let us consider a simply connected region $D$ with an arbitrary smooth boundary and corresponding complex plane $\omega$; with (2), Eq. (15) can be written as [Fig. 1(a)]

$$
2 \frac{\partial \tilde{e}}{\partial \omega}=2 Q \delta\left(x-x_{0}\right) \delta\left(y-y_{0}\right)+\frac{\partial \tilde{g}_{Z}}{\partial \omega} .
$$

According to Riemann's theorem [21] there exists a conformal mapping from the region $D$ to a circle in the corresponding $\chi$-plane: $\chi=\chi(\omega)$ with $\chi\left(\omega_{0}\right)=0$ $\left(\omega_{0}=x_{0}+i y_{0}\right)$, Fig. 1(a). By introducing the change of variables in (16), we arrive at

$$
2 \frac{\partial \tilde{e}}{\partial \chi} \frac{d \chi}{d \omega}=2 Q\left|\frac{d \chi}{d \omega}\right|^{2} \delta\left(x^{\prime}\right) \delta\left(y^{\prime}\right)+\frac{\partial \tilde{g}_{Z}}{\partial \chi} \frac{d \chi}{d \omega}
$$

Here $x^{\prime}$ and $y^{\prime}$ are defined as $\chi=x^{\prime}+i y^{\prime}$. Simplification of (17) gives:

$$
2 \frac{\partial \tilde{e}}{\partial \chi}=2 Q\left(\frac{d \chi}{d \omega}\right)^{*} \delta\left(x^{\prime}\right) \delta\left(y^{\prime}\right)+\frac{\partial \tilde{g}_{Z}}{\partial \chi} .
$$

From (18) one can see that the task of the deriving the field $e$ in the $D$ region could be reduced to the task of the deriving $e$ in a circle with the source with intensity $\left.2 Q\left(\frac{d \chi}{d \omega}\right)^{*}\right|_{\omega=\omega_{0}}$ placed in its center. Let us consider a circular contour $\Gamma$ in the $\chi$-plane such that $|\chi|=r<a$ ( $a$ is the radius of the circle). According to [21] along with Eq. (18), the contour integral over $\Gamma$ can be written as

$$
\oint_{\Gamma}\left(\tilde{e}^{*}-\frac{\tilde{g}_{Z}^{*}}{2}\right) d \chi=\left.2 i Q \frac{d \chi}{d \omega}\right|_{\omega=\omega_{0}} .
$$

Now let us rely on the fact that in case of a cylindrical structure with the particle traveling along the axis of the cylinder the longitudinal components (and also their $\zeta$ derivatives) of the electric and magnetic field $E_{z}, H_{z}$ do not depend on the transverse coordinates. In this case, in the $\chi$ plane from Eq. (14) we have

$$
\tilde{g}_{Z}=C \chi
$$

where $C$ is a constant. Because of the rotation symmetry in the $\chi$-plane the transverse field $e$ can be expressed as

$$
\tilde{e}=R(|\chi|) \frac{\chi}{|\chi|}
$$

Substituting (20) and (21) into (19) and taking into account that $R$ is a constant on $\Gamma$ one can write

$$
R \oint_{|\chi|=r} \frac{\chi^{*}}{|\chi|} d \chi-\frac{C^{*}}{2} \oint_{|\chi|=r} \chi^{*} d \chi=\left.2 i Q \frac{d \chi}{d \omega}\right|_{\omega=\omega_{0}}
$$

Evaluation of the integrals on the left side of (22) gives

$$
R=\left.\frac{Q}{\pi r} \frac{d \chi}{d \omega}\right|_{\omega=\omega_{0}}+\frac{C^{*}}{2} r
$$

Multiplication of $R$ by $\frac{\chi}{|x|}$ and taking into account that $|\chi|=r$ gives

$$
\tilde{e}=\left.\frac{Q}{\pi \chi^{*}} \frac{d \chi}{d \omega}\right|_{\omega=\omega_{0}}+\frac{C^{*} \chi}{2}
$$

Let us focus on the cross section that includes a particle. The idea of $[16,17]$ could be used to formulate a boundary condition at the point $\zeta=0$.

The electric and magnetic fields of the moving charge are nonzero inside the channel, but the field on the plane outside the channel for $z=c t$ vanishes [see Fig. 1(b)]. The field of the point particle on the plane vanishes because (1) the presence of retarding walls or layers outside the 
channel tenforce $V_{p h}<c$, and (2) the particle is ultrarelativistic, $\gamma \rightarrow \infty$. The combination of these two factors inevitably delays the electromagnetic field in the retarding media in the plane $\zeta=0(z=c t)$, moving with the particle. This allows the formulation of a boundary condition in the form

$$
\lim _{\zeta \rightarrow 0} e(|\chi|=a, \zeta)=0 .
$$

Using this condition in Eq. (24) we find

$$
C=-\left.\frac{2 Q}{\pi a^{2}}\left(\frac{d \chi}{d \omega}\right)^{*}\right|_{\omega=\omega_{0}} .
$$

and thus

$$
\tilde{e}=\left.\frac{Q}{\pi} \frac{d \chi}{d \omega}\right|_{\omega=\omega_{0}}\left(\frac{1}{\chi^{*}}-\frac{\chi}{a^{2}}\right) .
$$

At the point $\zeta=0$ application of the inverse Fourier transform yields

$$
\begin{aligned}
e(\zeta=0) & =\lim _{\zeta \rightarrow 0} \int_{-\infty}^{\infty} \exp (i k \zeta) \tilde{e} d k \\
& =\left.2 Q \delta(0) \frac{d \chi}{d \omega}\right|_{\omega=\omega_{0}}\left(\frac{1}{\chi^{*}}-\frac{\chi}{a^{2}}\right) .
\end{aligned}
$$

From (14), (18), and (26) we have

$$
\tilde{E}_{z}-i \tilde{H}_{z}=-\left.\frac{2 Q}{\pi i k a^{2}} \frac{d \chi}{d \omega}\left(\frac{d \chi}{d \omega}\right)^{*}\right|_{\omega=\omega_{0}} .
$$

Finally at the point $\zeta=0$ (the cross section that includes the particle) we have

$$
\begin{aligned}
E_{z}^{0}-i H_{z}^{0} & =-\left.\frac{2 Q}{a^{2}} \frac{d \chi}{d \omega}\left(\frac{d \chi}{d \omega}\right)^{*}\right|_{\omega=\omega_{0}} \lim _{\zeta \rightarrow 0} \int_{-\infty}^{\infty} \frac{\exp (i k \zeta)}{\pi i k} d k \\
& =-\left.\frac{2 Q}{a^{2}} \frac{d \chi}{d \omega}\left(\frac{d \chi}{d \omega}\right)^{*}\right|_{\omega=\omega_{0}} .
\end{aligned}
$$

If $\chi(\omega)=f\left(\omega, \omega_{0}\right)$ is the function that gives the mapping of the $D$ region onto a circle such that the point $\omega_{0}$ corresponds to the center of a circle we can write the result in a more compact form:

$$
\begin{aligned}
E_{z}^{0} & =-\frac{2 Q}{a^{2}} \operatorname{Re}\left[f^{\prime}\left(\omega, \omega_{0}\right)^{*} f^{\prime}\left(\omega_{0}, \omega_{0}\right)\right], \\
H_{z}^{0} & =-\frac{2 Q}{a^{2}} \operatorname{Im}\left[f^{\prime}\left(\omega, \omega_{0}\right)^{*} f^{\prime}\left(\omega_{0}, \omega_{0}\right)\right] .
\end{aligned}
$$

Formula (31) gives the values of the longitudinal field components $E_{z}$ and $H_{z}$ in the cross section of a particle which is moving along a vacuum channel of arbitrary shape. The formula is the two-dimensional Green function in the transverse coordinates. By integrating (31) over $\omega_{0}$ with the transverse distribution of a bunch $\rho_{\perp}\left(\omega_{0}\right)$, one can achieve fields for the bunch distributed in transverse coordinates.

According to the fundamental theorem of beam loading (see for example [22]) amplitude of the wake potential could be found though the longitudinal electric field $E_{z}^{0}$. Using this fact one can estimate the upper limit of a short bunch wakefunction. Formula for the longitudinal wake potential estimation is given in the Appendix A.

\section{TRANSVERSE PART OF THE LORENTZ FORCE}

We consider a part of the Maxwell system (3) and write it down in the expanded form:

$$
\begin{gathered}
\frac{\partial E_{z}}{\partial y}+\frac{\partial E_{y}}{\partial \zeta}=-\frac{\partial H_{x}}{\partial \zeta} \\
-\frac{\partial E_{x}}{\partial \zeta}-\frac{\partial E_{z}}{\partial x}=-\frac{\partial H_{y}}{\partial \zeta} \\
\frac{\partial H_{z}}{\partial y}+\frac{\partial H_{y}}{\partial \zeta}=\frac{\partial E_{x}}{\partial \zeta} \\
-\frac{\partial H_{x}}{\partial \zeta}-\frac{\partial H_{z}}{\partial x}=\frac{\partial E_{y}}{\partial \zeta}
\end{gathered}
$$

By combining (32),(33) and (34),(35) we have:

$$
\begin{aligned}
2 \frac{\partial}{\partial \zeta}\left(E_{x}-H_{y}\right) & =\frac{\partial H_{z}}{\partial y}-\frac{\partial E_{z}}{\partial x}, \\
2 \frac{\partial}{\partial \zeta}\left(E_{y}+H_{x}\right) & =-\frac{\partial H_{z}}{\partial x}-\frac{\partial E_{z}}{\partial y} .
\end{aligned}
$$

Using the definition of the Lorentz force acting on a particle we introduce a complex function $F_{\perp}$,

$$
F_{\perp}=q\left[E_{x}-H_{y}+i\left(E_{y}+H_{x}\right)\right],
$$

where $q$ is the charge of the test particle. From (36) and (37) we obtain

$$
2 \frac{\partial}{\partial \zeta}\left(F_{x}+i F_{y}\right)=q\left(\frac{\partial H_{z}}{\partial y}-\frac{\partial E_{z}}{\partial x}-i\left[\frac{\partial H_{z}}{\partial x}+\frac{\partial E_{z}}{\partial y}\right]\right) .
$$

In terms of the derivative (10) with respect to $\omega=x+i y$ (8) the equation above simplifies to:

$$
\frac{\partial F_{\perp}^{*}}{\partial \zeta}=-q \frac{\partial}{\partial \omega}\left(E_{z}-i H_{z}\right)
$$


In the vicinity of $\zeta=0$ with Eq. (29) we have

$$
F_{\perp}=\left.\frac{4 q Q \theta(\zeta) \zeta}{a^{2}}\left(\frac{d^{2} \chi}{d \omega^{2}}\right)^{*} \frac{d \chi}{d \omega}\right|_{\omega=\omega_{0}}
$$

Here $\theta(\zeta)$ is the Heaviside step-function. If $\chi(\omega)=$ $f\left(\omega, \omega_{0}\right)$ is the function that gives the mapping of the $D$ region onto a circle such that the point $\omega_{0}$ corresponds to the center of a circle we can write down the result in a more compact form

$$
F_{\perp}=\frac{4 q Q \theta(\zeta) \zeta}{a^{2}} f^{\prime \prime}\left(\omega, \omega_{0}\right)^{*} f^{\prime}\left(\omega_{0}, \omega_{0}\right)
$$

Formula (41) gives the values of the transverse part of the Lorentz force acting on a particle which is moving along a vacuum channel of arbitrary cross section. The formula is valid at and away from the particle and is the two-dimensional Green function. In the longitudinal coordinate, the formula is valid in the vicinity of the point $\zeta=0$. By integrating (41) over $\omega_{0}$ with the transverse distribution of the bunch $\rho_{\perp}\left(\omega_{0}\right)$ one can obtain the Lorentz force for the bunch distributed in the transverse coordinates.

\section{RELATIVISTIC GAUSS THEOREM}

In this section we will show how one can derive the relativistic Gauss theorem introduced in $[16,17]$ with the use of the formalism presented in Sec. II.

Consider Eq. (16) and integrate it over the vacuum gap cross section

$$
2 \int_{S_{\mathrm{vac}}} \frac{\partial \tilde{e}}{\partial \omega} d x d y=2 Q+\int_{S_{\mathrm{vac}}} \frac{\partial \tilde{g}_{Z}}{\partial \omega} d x d y
$$

and then evaluate the integral on the left-hand side according to Green's theorem

$$
\int_{S_{\mathrm{vac}}} \frac{\partial \tilde{e}}{\partial \omega} d x d y=\frac{i}{2} \oint_{\Gamma^{\prime}} \tilde{e} d \omega^{*}
$$

where $\Gamma^{\prime}$ is the boundary of the vacuum channel region $S_{\text {vac }}$.

Taking into account the boundary condition (25) $(e=0$ on $\Gamma^{\prime}$ ) with (14) we immediately obtain

$$
\int_{S_{\text {vac }}} E_{z} d S-i \int_{S_{\text {vac }}} H_{z} d S=-2 Q \lim _{\zeta \rightarrow 0^{+}} \int_{-\infty}^{\infty} \frac{\exp (i k \zeta)}{i k} d k .
$$

In the vicinity of the point $\zeta=0$ we have

$$
\int_{S_{\mathrm{vac}}} E_{z} d S-i \int_{S_{\mathrm{vac}}} H_{z} d S=-4 \pi Q \theta(\zeta),
$$

where $\theta(\zeta)$ is the Heaviside step function.

Expression (45) is a slightly generalized form of the relativistic Gauss theorem formulated in [16,17]. However, Eq. (45) states that an integral over the vacuum channel from the magnetic field $H_{z}$ is zero in the cross section of the particle and in a vicinity of the point $\zeta=0$. This result was not mentioned in [16].

Now, if one takes the point of the particle [which corresponds to $\omega=\omega_{0}$ in (31)], we arrive at the main result of the paper [16]

$$
E_{z}(0)=-\frac{2 Q}{a^{2}}\left|f^{\prime}\left(\omega_{0}, \omega_{0}\right)\right|^{2}=-\frac{2 Q}{a^{2}}|J|,
$$

where $|J|$ is the determinant of the Jacobi matrix at the point of the particle.

\section{SIMULATIONS AND RESULTS}

In this section we present results of calculations of the longitudinal electric field $E_{z}$ in the cross section of waveguides with the most common cross section shapes: cylindrical, planar, rectangular, and elliptical. The results were obtained by using formula (31) and found to be in full agreement with those obtained using the mode decomposition method $[14,23,24]$, where dielectric was considered as a retarding layer. For the case of the cylindrical structure we derived the two-dimensional Green function in explicit form. In Sec. VC we show how the suggested method can be combined with the image charge technique to derive the field in a cross section of a rectangular waveguide with retarding layers (up and down) and metal side walls. A rectangular waveguide with side metal walls is considered as an example, and a formula for the longitudinal electric field $E_{z}$ at the point of a particle (symmetrical case) is obtained. We also provide formulas for the loss factor and transverse $E_{z}$ field distribution for the case of an elliptical cross section (Sec. V D).

\section{A. Transverse distribution of the $E_{z}$ field in a cylindrical waveguide}

Conformal mapping of a circle with the radius $a$ onto a circle with radius $a$ such that the point $\omega_{0}=r_{0} \exp \left(i \phi_{0}\right)$ corresponds to the center of the second circle is given by

$$
f(\omega)=a^{2} \frac{\omega-r_{0} \exp \left(i \phi_{0}\right)}{a^{2}-\omega r_{0} \exp \left(-i \phi_{0}\right)},
$$

and its derivative by

$$
f^{\prime}(\omega)=\frac{a^{2}\left(a^{2}-r_{0}^{2}\right)}{\left[a^{2}-r_{0} \exp \left(-i \phi_{0}\right) \omega\right]^{2}} .
$$


Assuming $\omega=r \exp [\mathrm{i} \phi]$,

$$
f^{\prime}\left(\omega_{0}\right)=\frac{a^{2}}{a^{2}-r_{0}^{2}}
$$

and

$$
\operatorname{Re}\left[f^{\prime}(\omega)^{*}\right]=\frac{a^{2}\left(a^{2}-r_{0}^{2}\right)\left\{a^{4}-2 a^{2} r r_{0} \cos \left(\phi-\phi_{0}\right)+r^{2} r_{0}^{2} \cos \left[2\left(\phi-\phi_{0}\right)\right]\right\}}{\left[a^{4}+r^{2} r_{0}^{2}-2 a^{2} r r_{0} \cos \left(\phi-\phi_{0}\right)\right]^{2}}
$$

From (31) we have

$$
E_{z}=-\frac{2 Q}{a^{2}} \frac{a^{8}-2 a^{6} r r_{0} \cos \left(\phi-\phi_{0}\right)+r^{2} r_{0}^{2} a^{4} \cos \left[2\left(\phi-\phi_{0}\right)\right]}{\left[a^{4}+r^{2} r_{0}^{2}-2 a^{2} r r_{0} \cos \left(\phi-\phi_{0}\right)\right]^{2}} .
$$

For the comparison of present results with mode decomposition approach the method published in the paper [24] was used, where a circular dielectric loaded waveguide was considered. Comparison with [24] was made letting $\zeta=0$ in formulas published in [24] and particle energy $1 \mathrm{GeV}$. Table I contains a list of parameters for the cylindrical structure. Parameter $b$ corresponds to the outer radius of the waveguide and $\varepsilon$ is the dielectric constant of the layer. Figure 2 shows the angular dependence of the normalized longitudinal electric field $E_{z} /\left(-2 Q / a^{2}\right)$ for three different values of the test charge radial coordinate. We obtain full agreement with the mode decomposition method [24]. We should mention here that formula (51) is the twodimensional Green function for the cylindrical structure.

\section{B. Transverse distribution of the $E_{z}$ field in a planar waveguide}

Conformal mapping of a strip with the half-width $a$ onto a circle with the radius $a$ such that the point $\omega_{0}=0$ corresponds to the center of the circle is given by

$$
f(\omega)=a \tan \left[\frac{\pi \omega}{4 a}\right]
$$

and its derivative by

$$
f^{\prime}(\omega)=\frac{\pi}{4}\left(\sec \left[\frac{\pi \omega}{4 a}\right]\right)^{2}
$$

TABLE I. Parameters for the cylindrical structure.

\begin{tabular}{lcccc}
\hline \hline$a$ & $b$ & $\varepsilon$ & $r_{0}$ & $Q$ \\
\hline $0.3 \mathrm{~cm}$ & $0.32 \mathrm{~cm}$ & 5.7 & $0.15 \mathrm{~cm}$ & $1 \mathrm{nC}$ \\
\hline \hline
\end{tabular}

Assuming $\omega=x+i y$,

$$
f^{\prime}\left(\omega_{0}\right)=\frac{\pi}{4}
$$

and

$$
\operatorname{Re}\left[f^{\prime}(\omega)^{*}\right]=\frac{\pi}{2} \frac{1+\cos \left[\frac{\pi x}{2 a}\right] \cosh \left[\frac{\pi y}{2 a}\right]}{\left(\cos \left[\frac{\pi x}{2 a}\right]+\cosh \left[\frac{\pi y}{2 a}\right]\right)^{2}} .
$$

From (31) we then have

$$
E_{z}=-\frac{2 Q}{a^{2}} \frac{\pi^{2}}{8} \frac{1+\cos \left[\frac{\pi x}{2 a}\right] \cosh \left[\frac{\pi y}{2 a}\right]}{\left(\cos \left[\frac{\pi x}{2 a}\right]+\cosh \left[\frac{\pi y}{2 a}\right]\right)^{2}} .
$$

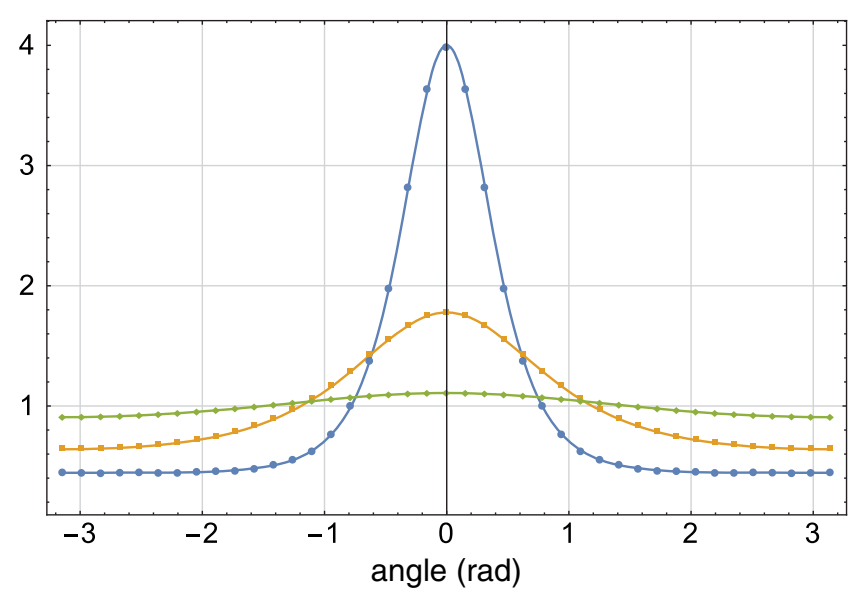

FIG. 2. Dependence of $E_{z}$ on azimuthal angle $\phi-\phi_{0}$ normalized by a factor of $-2 Q / a^{2}$ for the case of an off axis displaced particle. Orange line: $r=r_{0}$; Green line: $r=a / 10$; Blue line: $r=a$. Points are results for the direct simulation using the mode decomposition method [23,24] (1000 radial and 15 azimuthal modes were taken in to account) and the solid lines are calculated using the formula (51). 
TABLE II. Parameters for the planar structure.

\begin{tabular}{lcccc}
\hline \hline$a$ & $b$ & $\varepsilon$ & $r_{0}$ & $Q$ \\
\hline $0.5 \mathrm{~cm}$ & $0.502 \mathrm{~cm}$ & 5.7 & $0 \mathrm{~cm}$ & $1 \mathrm{nC}$ \\
\hline \hline
\end{tabular}

For the comparison of present results with mode decomposition approach the method published in [14] was used, where a rectangular waveguide with two parallel dielectric layers was considered. Comparison with [14] was made letting $\zeta=0$ in formulas published in [14] and particle energy $1 \mathrm{GeV}$. Table II contains the parameters for the planar structure. Parameter $b$ corresponds to the distance from the center of the structure to the metal wall and $\varepsilon$ is the dielectric constant of the layer; height of the waveguide (parameter $w$ in [14]) was take $10 \mathrm{~cm}$; parameter $r_{0}$ is the displacement of the particle from the center of the structure. Figure 3 shows the coordinate dependence of the longitudinal electric field $E_{z}$ normalized by $-2 Q / a^{2} \times \pi^{2} / 8$ on the $x$ and $y$ coordinates. We see full agreement with the mode decomposition method [14]. Note that originally formula (56) was obtained in [25] and later in [26], where it was derived using field matching methods and assuming the impedance can be characterized as a surface impedance. We should mention that equation (56) could not be treated as a Green function as it does not depend on the coordinates of the source particle.

\section{Longitudinal electric field $E_{z}$ of a particle in a rectangular structure with metal side walls}

We consider a rectangular structure which differs from the planar structure by the presence of two parallel perfect conducting walls in the $y-z$ plane. In the case where the field distribution in the vacuum gap is known for the planar case (56), the contribution of the metal walls can be included by introducing image charges. If the field for the planar case is $E_{z}^{p l}(x, y)$ and metal walls are placed at $y= \pm w / 2$, the full field can be found as a superposition of the field of real charge field and imaginary charges. In the case where the charge is placed in the center of the structure, the full field is given by

$$
\begin{aligned}
E_{z}^{\mathrm{rec}}(x, y)= & E_{z}^{\mathrm{pl}}(x, y)+\sum_{n=1}^{\infty}(-1)^{n} E_{z}^{\mathrm{pl}}(x, y-n w) \\
& +\sum_{n=1}^{\infty}(-1)^{n} E_{z}^{\mathrm{pl}}(x, y+n w) .
\end{aligned}
$$

The longitudinal electric field $E_{z}$ at the particle is then

$$
E_{z}^{\mathrm{rec}}(0,0)=E_{z}^{\mathrm{pl}}(0,0)+2 \sum_{n=1}^{\infty}(-1)^{n} E_{z}^{\mathrm{pl}}(0, n w) .
$$

By substituting (56) into (58), we obtain

$$
E_{z}^{\mathrm{rec}}(0,0)=-\frac{2 Q}{a^{2}} \frac{\pi^{2}}{16}\left[1+4 \sum_{n=1}^{\infty} \frac{(-1)^{n}}{1+\cosh \left(\frac{\pi n w}{2 a}\right)}\right] .
$$

Figure 4 shows the dependence of the normalized longitudinal electric field $E_{z} /\left(-2 Q \pi^{2} / 16 a^{2}\right)$ at the particle on the relative width of the waveguide $a / w$. Full agreement with the mode decomposition method [14] is obtained. Comparison with [14] was made letting $\zeta=0$ in formulas published in [14] and particle energy $1 \mathrm{GeV}$. Waveguide parameters are listed in Table II.

\section{Transverse distribution of the $E_{z}$ field in an elliptical waveguide}

Consider an elliptic cross section with semimajor axis $b$ and semiminor axis $a$ and the particle located in the center of this ellipse. Then according to [27] the conformal mapping of an ellipse with these semiaxes onto a circle of a radius $a$ with the center of the ellipse corresponding to the center of a circle is given by
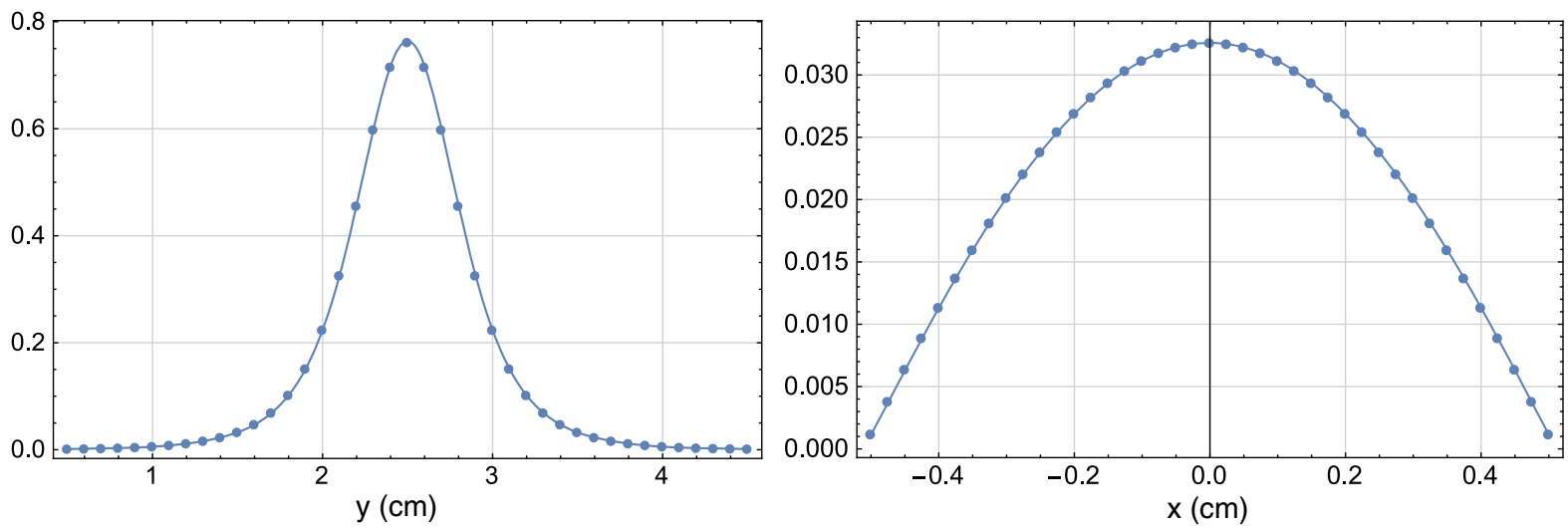

FIG. 3. Y-dependence (left) and X-dependence (right) of $E_{z}$ normalized by $-2 Q / a^{2} \times \pi^{2} / 8$ in the case where the test charge is located at $x=0.4 \mathrm{~cm}$ from the center for the $\mathrm{y}$-plot (left) and $y=3.8 \mathrm{~cm}$ for the $\mathrm{x}$-plot (right). Points are computed using the mode decomposition method [14] (array of 5 by 500 modes were taken into account) and solid lines are obtained from formula (56). 


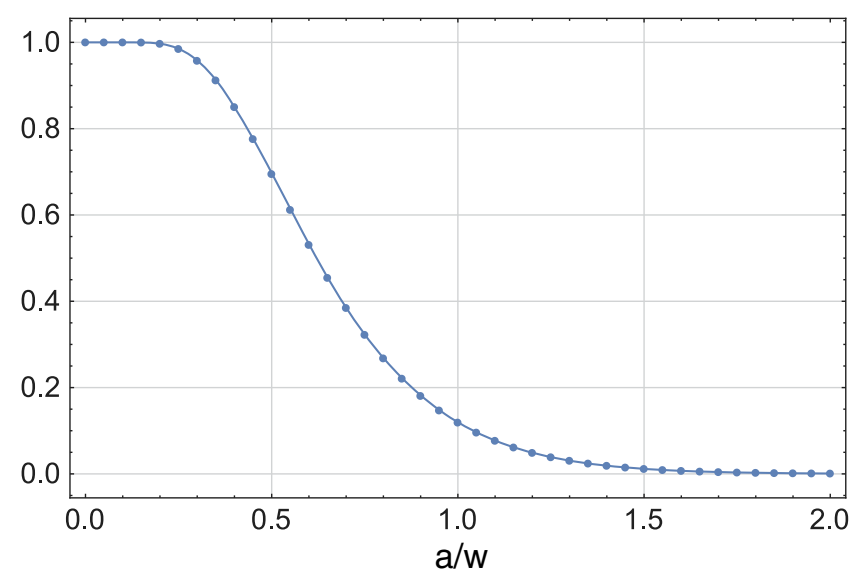

FIG. 4. Dependence on the waveguide relative width $a / w$ of the normalized longitudinal electric field $E_{z}$ at the particle in a rectangular waveguide. Solid curve: dependence of formula (59) on $a / w$ normalized by $-\frac{2 Q}{a^{2}} \frac{\pi^{2}}{16}$; points: results using the mode decomposition method [14] and also normalized by $-\frac{2 Q}{a^{2}} \frac{\pi^{2}}{16}$.

$$
f(\omega)=a \sqrt{k} \operatorname{sn}\left[\frac{2 \mathrm{~K}}{\pi} \arcsin \left(\frac{\omega}{\sqrt{b^{2}-a^{2}}}\right), k^{2}\right],
$$

where

$$
k=4 \sqrt{q}\left(\prod_{n=0}^{\infty} \frac{1+q^{2 n}}{1+q^{2 n+1}}\right)^{4}
$$

and

$$
q=\left(\frac{b-a}{b+a}\right)^{2}
$$

Here $\operatorname{sn}\left(z, k^{2}\right)$ is the Jacobi elliptic sine function with elliptic modulus $k$, and $\mathrm{K}$ is the complete elliptic integral of the first kind

$$
\mathrm{K}\left(k^{2}\right)=\int_{0}^{1} \frac{d t}{\sqrt{\left(1-t^{2}\right)\left(1-k^{2} t^{2}\right)}} .
$$

Using (31) one can write the formula for the longitudinal component of the electric field $E_{z}$ as

$$
\begin{aligned}
E_{z}= & -\frac{2 Q}{a^{2}} \frac{4 a^{2} k \mathrm{~K}^{2}}{\pi^{2} \sqrt{b^{2}-a^{2}}} \\
& \times \operatorname{Re}\left\{\frac{\operatorname{cn}\left[\frac{2 \mathrm{~K}}{\pi} \mathrm{acrsin}\left(\frac{x+i y}{\sqrt{b^{2}-a^{2}}}\right), k^{2}\right] \operatorname{dn}\left[\frac{2 \mathrm{~K}}{\pi} \mathrm{acrsin}\left(\frac{x+i y}{\sqrt{b^{2}-a^{2}}}\right), k^{2}\right]}{\sqrt{b^{2}-a^{2}-(x+i y)^{2}}}\right\} .
\end{aligned}
$$

At the particle location $x=0, y=0$ we have

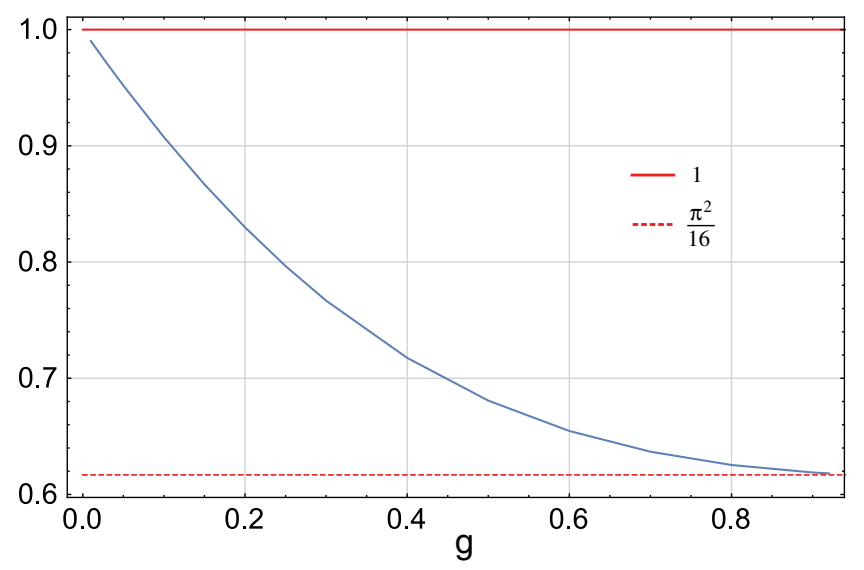

FIG. 5. Longitudinal electric field $E_{z}$ at the particle in the elliptical structure normalized by $-\frac{2 Q}{a^{2}}$, showing its dependence on the ellipse flattering factor $g=1-a / b$.

$$
E_{z}=-\frac{2 Q}{a^{2}} \frac{4 a^{2} k \mathrm{~K}^{2}}{\pi^{2}\left(b^{2}-a^{2}\right)}
$$

Figure 5 shows the dependence of the fields $E_{z}$ (63) on the ellipse flattering factor $g=1-a / b$. As expected, if $g \rightarrow 1$ the form factor approaches that of the planar waveguide $\pi^{2} / 16$ and in the case $g \rightarrow 0$ the from factor approaches unity.

The $E_{z}$ field distribution results presented above correspond to the central position of the particle, $x=0, y=0$ and could not be treated as a Green function. If the particle passes through the structure with some offset, the same type of formula can be obtained following the procedure described in Sec. VA for the circular waveguide with the help of a formula (31).

The elliptical shape considered in many publications previously using the mode decomposition approach required numerical modeling, and a significant number of modes need to be taken in account, especially for an observation point close to the structure surface. As a result, the usual approach for this type of geometry is approximation of a circular waveguide for the aspect ratio $a / b \sim 1$ and a planar structure for $a / b \sim 0$ accordingly. In many cases, however, aspect ratios in the range of $a / b \sim 0.1-0.83$ need to be considered. For example, the FERMI@Elettra FEL undulator designs use [15] aspect ratios of 0.17-0.83 corresponding to flatness factors $g \sim 0.83-0.17$. Formula (62) (Fig. 5) gives rigorous analytical solutions for the $E_{z}$ field for this range of parameters. It should be noticed that if a circular geometry approximation is used the relative error for the $a / b<0.83$ aspect ratio $(g>0.17)$ exceeds $15 \%$ for the fixed chamber gap $(r=a)$ while the planar geometry approximation error is above $1 \%$ for a flatness factor $g<0.83(a / b>0.17)$. The corresponding circular waveguide approximation does not look like a solution for any noticeable elliptical deformation because of the sharp increase in the form-factor relative error 
(Fig. 5). Note that the $E_{z}$ field for the displaced beam can be also obtained with analytical expressions following the same procedure as presented in Sec. VA for circular cross sections and formula (31). Finally, formula (63) is a simple analytical expression for $E_{z}$ components of the wakefield generated by a point particle passing arbitrary elliptical shape waveguide. This allows a simple method to be used to calculate the relative error of using a circular or planar approximation for elliptical undulators like LCLS-II [28] (planar approximation), NSLS-II $[29,30]$ (aspect ratio $a / b \sim 0.3)$, SXFEL [31] $(a / b=0.4)$, CLARA FEL [32] $(a / b=0.3)$, European X-FEL [33] $(a / b \sim 0.5)$, and CLIO FEL [34] $(a / b \sim 0.4)$.

\section{CONCLUSION}

In this paper we have derived a general formula for the longitudinal electromagnetic field components of a point charged particle passing the arbitrary shape waveguide. The derived expressions for the fields are valid at the cross section of the particle. The waveguides are considered to be longitudinally homogeneous with arbitrary retarding layers. The obtained results can be used for the exact calculations of the upper limit (peak amplitude) of the loss factors and wake potentials. The formula expresses the field though the derivative of the function that conformally maps the channel of the form one is interested in onto a circle. It is worth mentioning that the mapping function itself does not appear in the formula and one needs to know only its derivative. We also obtained a formula for the transverse (deflecting) Lorentz force, which can be used for the transverse wake potential calculations for the bunches with extended transverse distributions. As an example of the application of this formalism we have derived the explicit form of the two-dimensional Green function for the $E_{z}$ field for the case of a cylindrical structure. We have also derived the $E_{z}$ field at the point of and away from a particle driving along the axis of a planar structure, of a rectangular structure with metal side walls and of an elliptical structure. The developed formalism represents a new powerful analytical approach in wakefield calculations for short bunches in FELs and Linear Collider.

\section{ACKNOWLEDGMENTS}

The authors are grateful to P. Schoessow for useful discussions and suggestions. This work was partially supported by Russian Foundation For Basic Research Grant No. 15-02-08745A.

\section{APPENDIX A: SHORT RANGE LONGITUDINAL WAKEFUNCTION ESTIMATION}

In this appendix we show how the developed formalism can be applied to estimate longitudinal short range wakefunction.
According to the fundamental theorem of beam loading (see for example [22]) longitudinal wake potential right after the point particle equals twice the value of the wake potential at the point particle

$$
w_{\|}\left(0^{+}\right)=2 w_{\|}(0) .
$$

For the longitudinally homogeneous structure longitudinal wake potential could be expressed through the longitudinal component of the electric field $E_{z}$ as

$$
w_{\|}(\zeta)=-\frac{E_{z}(\zeta)}{Q} L
$$

where $L$ is the length of the structure. Combining (31), (A1), and (A2) we get

$$
w_{\|}\left(0^{+}\right)=\frac{4 L}{a^{2}} \operatorname{Re}\left[f^{\prime}\left(\omega, \omega_{0}\right)^{*} f^{\prime}\left(\omega_{0}, \omega_{0}\right)\right] .
$$

Wakefunction decays over the distance. For the most cases the condition

$$
w_{\|}\left(0^{+}\right) \geq w_{\|}(\zeta)
$$

holds. This leads to an estimation of the short range wake potential of a bunch with longitudinal distribution $\rho_{z}(\zeta)$ in the form

$$
W_{\|}(\zeta) \leq w_{\|}\left(0^{+}\right) \int_{-\infty}^{\zeta} \rho_{z}\left(\zeta_{0}\right) d \zeta_{0}
$$

Substitution of (A3) into (A5) gives the longitudinal wake potential estimation for the short bunch

$W_{\|}(\zeta) \leq \frac{4 L}{a^{2}} \operatorname{Re}\left[f^{\prime}\left(\omega, \omega_{0}\right)^{*} f^{\prime}\left(\omega_{0}, \omega_{0}\right)\right] \int_{-\infty}^{\zeta} \rho_{z}\left(\zeta_{0}\right) d \zeta_{0}$.

Formula (A6) should be treated as the upper limit of the wakefunction, direct application may lead to the overestimation of the wake. For example in [26] application of such approximation to the calculation of the loss factor of a $30 \mu \mathrm{m}$ uniform bunch in a planar corrugated RadiaBeam/LCLS dechirper showed 20-30\% overestimation in comparison with numerical simulation results.

\section{APPENDIX B: SHORT RANGE TRANSVERSE WAKEFUNCTION ESTIMATION}

In contrast to longitudinal wake potential inequality

$$
w_{\perp}\left(0^{+}\right) \geq w_{\perp}(\zeta)
$$


yet to be proved. However we believe that for the case of a short bunch one can still estimate upper boundary of the transverse wake potential as

$$
W_{\perp}(\zeta) \leq w_{\perp}\left(0^{+}\right) \int_{-\infty}^{\zeta}\left(\zeta-\zeta_{0}\right) \rho_{z}\left(\zeta_{0}\right) d \zeta_{0}
$$

In [26] it was shown for the case of a planar structure that numerically obtained transverse wake potential was found to be $20-30 \%$ less than its estimated value.

With (41) taking into account

$$
w_{\perp}(\zeta)=L \frac{F_{\perp}(\zeta)}{q Q},
$$

we get

$W_{\perp}(\zeta) \leq \frac{4 L}{a^{2}} f^{\prime \prime}\left(\omega, \omega_{0}\right)^{*} f^{\prime}\left(\omega_{0}, \omega_{0}\right) \int_{-\infty}^{\zeta}\left(\zeta-\zeta_{0}\right) \rho_{z}\left(\zeta_{0}\right) d \zeta_{0}$.

[1] ILC Technical Design Report, Volume 1: Executive Summary, 2013.

[2] M. Aicheler. A Multi-TeV Linear Collider Based on CLIC Technology: CLIC Conceptual Design Report (unpublished).

[3] C. Bostedt et al., J. Phys. B 46, 164003 (2013).

[4] J. Galayda et al., LCLS-II Conceptual Design Report, SLAC Report: SLAC-R-978, 2011.

[5] W. A. Barletta et al., Nucl. Instrum. Methods Phys. Res., Sect. A 618, 69 (2010).

[6] M. Byrd, T. J. Shea, P. Denes, P. Siddons, D. Attwood, F. Kaertner, L. Moog, Y. Li, A. Sakdinawat, and R. Schlueter, Nucl. Instrum. Methods Phys. Res., Sect. A 623, 910 (2010).

[7] K. F. Bane, Report No. SLAC-Pub-11829, 2006.

[8] K. F. Bane and G. Stupakov, Nucl. Instrum. Methods Phys. Res., Sect. A 690, 106 (2012).

[9] A. Novokhatski and A. Mosnier, in Proceedings of the Particle Accelerator Conference, Vancouver, BC, Canada, 1997 (IEEE, New York, 1997), p. 1661.

[10] K. L. F. Bane and G. Stupakov, Phys. Rev. ST Accel. Beams 6, 024401 (2003).
[11] A. Chao, in Physics of Collective Beam Instabilities in High Energy Accelerators (Wiley and Sons, New York, 1993).

[12] S. Y. Park, C. Wang, and J. L. Hirshfield, AIP Conf. Proc. 647, 527 (2002).

[13] Changbiao Wang and J. L. Hirshfield, Phys. Rev. ST Accel. Beams 9, 031301 (2006).

[14] S. S. Baturin, I. L. Sheinman, A. M. Altmark, and A. D. Kanareykin, Phys. Rev. ST Accel. Beams 16, 051302 (2013).

[15] A. Lutman, R. Vescovo, and P. Craievich, Phys. Rev. ST Accel. Beams 11, 074401 (2008).

[16] S. S. Baturin and A. D. Kanareykin, Phys. Rev. Lett. 113, 214801 (2014).

[17] S. S. Baturin and A. D. Kanareykin, arXiv:1409.0209.

[18] S. S. Baturin and A. D. Kanareykin, arXiv:1308.6228.

[19] B. Zotter and S. Kheifets, Impedances and Wakes in High Energy Particle Accelerators (World Scientific, Singapore, 1998).

[20] P. M. Morse and H. Feshbach, Methods of Theoretical Physics. Part I. (McGraw-Hill Science/Engineering/Math, New York, 1953), Ch. 4, p. 330.

[21] M. A. Lavrentiev and B. V. Shabat, Methods of Complex Function Theory (Nauka, Moskow, 1987).

[22] P. B. Willson, Report No. SLAC-PUB-4547, 1989.

[23] K.-Y. Ng, Phys. Rev. D 42, 1819 (1990).

[24] A. M. Altmark and A. D. Kanareykin, J. Phys. Conf. Ser. 357, 012001 (2012).

[25] S. S. Baturin, arXiv:1507.03901.

[26] K. Bane and G. Stupakov, arXiv:1601.07226.

[27] G. Valiron, Fonctions Analytiques (Press Universitaires de France, Paris, 1954).

[28] K. L. F. Bane and G. Stupakov, Report No. SLAC-PUB10707, 2004.

[29] A. Blednykh and S. Krinsky, in Proceedings of the 22nd Particle Accelerator Conference, PAC-2007, Albuquerque, NM (IEEE, New York, 2007), p. 4321.

[30] C. Hetzel et al., in Proceedings of the 3rd International Particle Accelerator Conference, New Orleans, LA, 2012 (IEEE, Piscataway, NJ, 2012), p. 2558.

[31] M. Song et al., arXiv:1512.05462.

[32] S. Spampinati et al., in Proceedings of 5th International Particle Accelerator Conference (IPAC 2014), Dresden, Germany (CERN, JACoW conferences, Geneva, Switzerland, 2014), p. 2930.

[33] M. Dohlus, T. Limberg, and I. Zagorodnov, TESLA-FEL Report 2005-10, 2005.

[34] Hole Coupled Infrared Free-Electron Laser, Free Electron Lasers, edited by Dr. Sandor Varro (InTech, Prazeres Rui, 2012), ISBN: 978-953-51-0279-3. 